\title{
Cloud Based Electronic Program Guide System with Integrated Social Network Recommendations
}

\author{
Emad Al-Mohammed*, Nigel Linge \\ School of Computing, Science and Engineering, University of Salford, Manchester, United Kingdom. \\ * Corresponding author. Tel.: 00-44-7412702306; email: e.a.almohammed@edu.salford.ac.uk \\ Manuscript submitted October 3, 2016; accepted December 1, 2016. \\ doi: 10.17706/ijeeee.2017.7.2.100-110
}

\begin{abstract}
Television viewing is changing with a growing trend towards online consumption of content with a proliferation of providers and an increasing range of viewing devices available. This trend will start to challenge the future of the traditional television channel as more and more viewers becoming focused on the programs, rather than the broadcaster or provider who distributes them. However, this in turn poses a problem for the viewer in that it will become increasingly difficult to locate those programs of interest across such a broad range of providers. This paper introduces a new architecture for a generic cloud based Electronic Program Guide (EPG) that can access all of the available content sources. The architecture of the generic EPG system allows for program metadata to be retrieved from online providers in a single search operation despite the difference in metadata formats that are supported by the different providers. Moreover, this system provides a personalized EPG that takes the program as its core focus and outputs a list of recommendations that are based on the viewer's interests, viewing pattern and the recommendations of their friends from within the social networks they belong to. Additionally, the system responds to the dynamic environment features such as viewing device, network bandwidth, and service registrations to ensure that any recommended program can indeed be watched by the viewer at that particular time.
\end{abstract}

Key words: Online TV, personalized electronic program guide, social networks, recommendation system.

\section{Introduction}

Nowadays, watching television has become a daily part of our digital lives, especially with the growth in the range of content sources available. This has seen a switch from traditional linear viewing of broadcast content to time shifted or on-demand online viewing through providers such as YouTube, Amazon and Netflix. This has led the viewer to move from the television as device towards other types of devices such as personal computers, smartphones or tablets especially in the younger age groups. That is clear from the growth in video streaming traffic which has been reported by the network providers of $4 \mathrm{G}$ mobile services [1], [2]. Additionally, the variety in the content sources brings another challenge which is how to connect with all of these sources and search them for a particular list of programs.

Moreover, creating such a list of programs for each user that represents their interests and matches their viewing habits becomes increasingly difficult. On the other hands, social networks have started to dominate the ways friends and family members contact each other. Its influence doesn't impact only the relationships between people, but it has become a method to share opinions, choices and experience between the network's members [3], [4]. Consequently, integrating social networks into a recommendation scheme can enhance the recommendation process and provide a broader choice for users especially when considering 
television viewing.

Viewers want to seek content based on the programs that they want to watch regardless of their providers. Current Electronic Program Guide (EPG) systems tend to be related to one provider or set of providers requiring the viewer to have to use several different EPG systems in order to search the complete range of content sources that are now available to them. Consequently, it is important for a single EPG to be developed that is able to search all of the available providers and online sources in one personalized search to offer a single list of favourite programs.

In order to address these challenges, we have developed a new generic cloud based EPG with an architecture that can interface with any provider of online television. The EPG is delivered as a cloud based service from a server to which users must create an account. The user's preferences and historical data are stored within this server which is also connected to providers of online content. Programs of potential interest will be retrieved from all these available online content sources in a single search operation. Such a system will then be able to recommend the programs based on users' viewing history, viewing pattern and user context including the time of day, network available bandwidth and the device that is used to view the television content. Moreover, social networks can be included and exploited to enhance the recommendation process, where the system can interact with the user friends' and family members' accounts to offer further recommendations based on their interests.

This paper presents an overview of the new EPG system architecture that has been developed and focuses on the challenges of connecting with the different video content sources and how the recommendation system, depending on the retrieved programs' metadata, can recommend a personalized list of programs that match the user interests and his friends recommendations.

\section{Related Works}

\subsection{Multiple Video Content Sources}

In order to design an integrated EPG system, it is very important to design a searching system that has the ability to communicate with different content sources. That is because each provider offers content using their own metadata format. Therefore, multiple search engines should be used to search those providers with each search engine passing query terms through the Application Programming Interface offered by that provider [5]. Since those providers adopt a different format for their information, it has necessitated the translation of a content provider's metadata to a uniform format used within the EPG. Posoldova described how to collect the data from two different sources (TV and Internet) by indicating the global effects and translating the data format in order to use this information in television recommendation system [6].

Rothschild proposed a searching system that integrates a TV with Internet content. This is based on searching the Internet for the viewed program depending on one of the parameters that describes the TV programs such as title or genre. The result of the searching operation could then be a document or video or web site that contains something relevant [7]. A new searching system has been proposed by Kim for Smart TVs based on multi-level searching on three multimedia content sources. Users need to enter the query keywords using the Smart TV browser, and then the browser will send these keywords to the searching system to search a local repository for matching programs. The same keywords will be sent to another searching level where the EPG repository and global repository will be searched to find matching programs [8].

However, our cloud-based EPG system depends on multiple search agents controlled by search agent leader. Each agent has the ability to interpret the metadata that retrieved from specific video content source into a unified standard format which is used within the processing and recommendation operations. 


\subsection{Recommendation Systems}

Recommendation systems have had significant attention by researchers due to the importance of these systems in many applications. Several solutions have been proposed by the researchers for recommendation systems; each one of them uses a particular technique to recommend items to users. One of the recommendation techniques is a content-based approach which means recommending items that have a high similarity degree with the items that have been highly rated by the user. Another approach is the collaborative filtering approach which is based on clustering users into particular groups depending on similar selected items or programs. Then, it recommends items which are missing from the target user profile but that have been watched by the others [9], [10]. However, those approaches have several drawbacks such as over-specialize and cold-start problems. To eliminate those drawbacks, a hybrid technique has been proposed by combining both the content-based and collaborative filtering techniques to enhance the recommended programs within an EPG [11].

Another technique that has been presented by Song where the user context is taken into account. There are many types of context such as user context, device context, network context, content context and dynamic context [12]. For more personalization, several researches designed recommendation systems to include more than one recommendation technique. They depend on: an explicit profile which contains information relevant to each user, an implicit profile based on a statistical analysis for the collected information that is relevant to the user's viewing history, user ratings for watched programs and user context [13], [14].

However, our system designed to include the traditional recommendation techniques and took the effects of the current environment features and user context such as time of day, user's device type and available network bandwidth. Moreover, the social networks have been included to enhancing the recommendation process based on the closed friends' and family members' recommendations.

\subsection{Social Networks}

A popular way to connect with friends and family is through social networks. Social networks are used not only to communicate with each other, but also to exchange ideas and interests through sharing, inviting and recommending. Demographic information, such as age and gender, is one of the factors that impacts the similarity of users' interests especially in respect of TV programs and music. Researches have showed that people express similar choices and interests in the domains of movies, TV shows and music when they have similar demographic information or they are friends or they share more than one common friend. Consequently, social networks increase the interaction between people and enhance the sense of community [15]. Due to the growing importance of social networks, many studies have been focused on the nature of relationships between users and how these relationships can affect the selections and help users to make decisions. For this reason, social recommendation has been investigated on the basis of psychology and sociology studies. Two factors have been specified as social context which are individual preferences and interpersonal influences. Jiang discussed the effects of the social relationships on users' selections and their interaction with the recommendation systems. The results showed that the recommendation performance has been improved because of this social influence [16].

A study has demonstrated how using Facebook as a source of information can improve the recommendation process when there is a lack of information for new users or sparse data. This can be a good solution that overcomes the drawbacks of other recommendation techniques such as collaborative filtering [17]. Another proposal presented by Quijano-Sanchez includes creating an application within Facebook to recommend movies and TV programs between the users who join this application [18]. However, this application supports the user with recommendations within the Facebook and never used 
this information to support an EPG system externally. Our system exploits the social network recommendations (as example Facebook) by retrieving the friends' and family members' recommendation and translating this information into a unified standard format then using this information to enhance the recommendation process within the entire system.

\section{System Design and Implementation}

Our new generic EPG system operates as a central cloud base service. It provides the EPG as web page and the users need to create an account and login in order to use the system recommendation services. The system design allows the users to search any program across any number of online television providers. Except for the searching agents that interface with each different content provider, the architecture of the system is generic. The different metadata formats used by content providers is converted into a single format which is used internally in the remainder of the system. The recommendation part employs three traditional recommendation techniques: content-based, collaborative filtering and context-awareness but additionally, the system exploits the social networks to enhance the recommendation process. The recommended programs are then filtered based on user context to match the device that the user is currently using.

\subsection{Multiple Sources Search System}

The searching system is the most important part of any EPG system. Our system includes multiple searching agents, each one of them connected with one provider of online content. These searching agents are analogous to the television tuner because it allows the system to communicate with the different providers and translate their information into a unified internal format as shown in Fig. 1. The system requires an interface with each online content provider to retrieve the program metadata which is provided by each source. Due to the different formats of metadata which is provided by each content source, each search agent has an interpreter to unify these metadata into unified format to use within the EPG system as shown. These searching agents are controlled by a search agent leader which controls the searching operation and prepares the retrieved metadata for the next steps. The searching operation is based on keywords where the system will search the programs that have similarity with those keywords. The search agent leader controls all of the searching operations such as searching for the programs that have similarity with those that have been watched before, those which are recommended by the system based on the clustering approach or social networks and those are searched directly based on keywords provided by the user. Thereafter, the search results are combined and parsed to remove the duplicates and the associated metadata is saved in the content database server.

Currently, the system has been implemented to access the following online content sources: BBC-iPlayer, YouTube, Dailymotion and The Movie Database (TMDb). BBC-iPlayer provides its metadata files in XML while the others provide their metadata files in JSON. In order to retrieve the metadata from these various content sources, a search agent module communicates and fetches the required metadata from given source and translates and saves the results in XML format which is subsequently used by the functions which control the analyzing operations. Then the search agent leader reads those files and creates a programs matrix. Each matrix includes 22 program features such as title, synopsis, name of leading actor, genre, type, date of production, language, subtitle, duration, director, ratings, resolution, format, subtitle, cast, producer name, author, country, age, gender, parental level, availability and address.

These searching operations (except the one which is applied directly using keywords provided by the user) are implemented when the user is not actively using the EPG (logged off). The only operation that will be implemented when the user logs in to the EPG is the filtering of the programs list based on the features of the user's viewing environment. This will decrease the time needed for recommending the programs and 
improve the user experience.

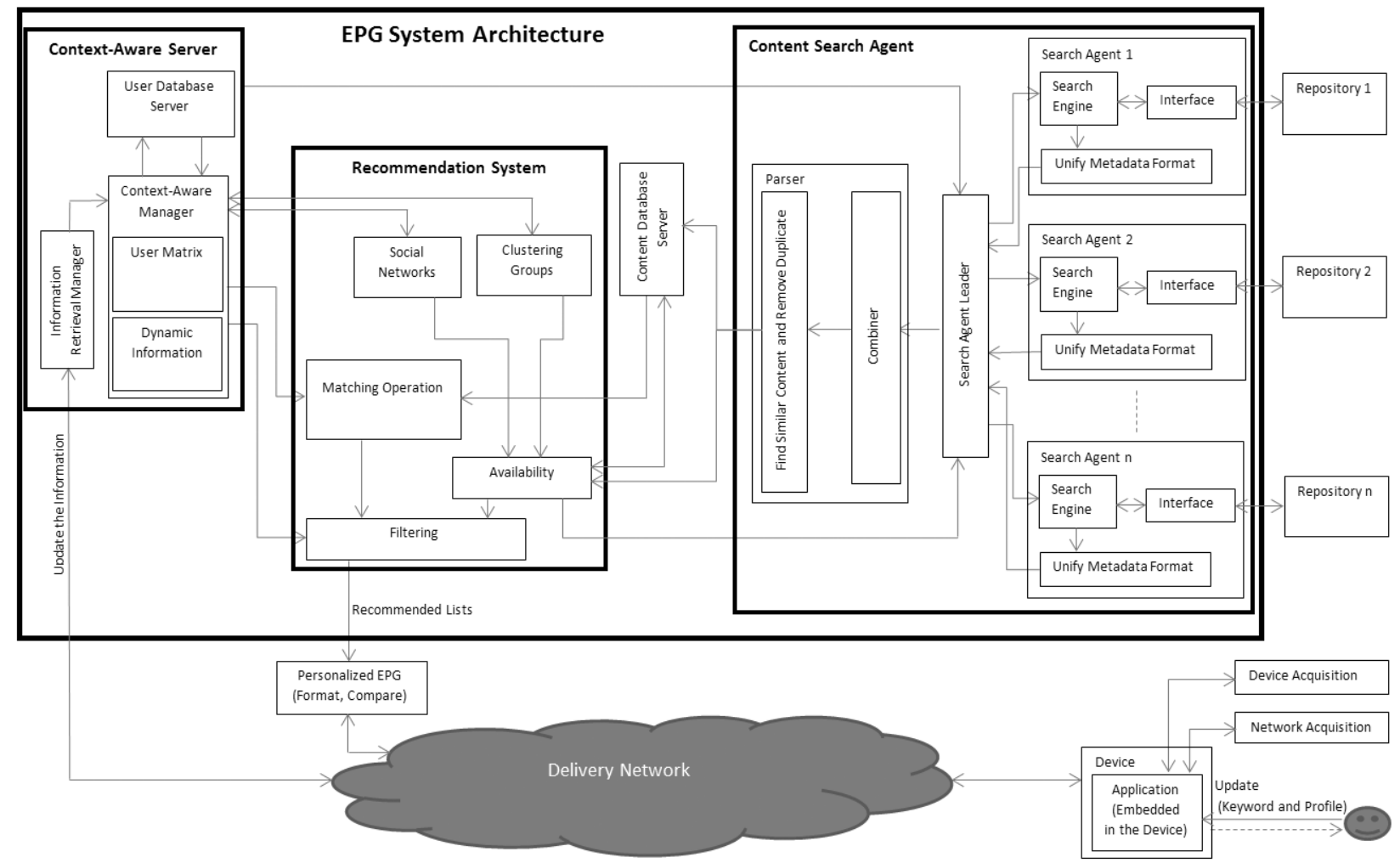

Fig. 1. Overall system configuration.

\subsection{Recommendation System}

The recommendation part of the EPG system is responsible for recommending a personalized list of programs that is better tailored with users' interests. Basically, three lists of programs are created based on the recommendation approaches which are used. The first list is generated based on the user viewing history where the titles of the watched programs have been sent to the search agents' leader to search the different content sources for similar programs. Then, the retrieved program's metadata will be translated to the unified format and saved in the content database server (see Fig. 1). Thereafter, a list of programs is generated based on the viewing history of each hour. The viewing history of each user is divided into 24 parts with each representing one hour of day. Similarity calculations are carried out using the cosine similarity to find the higher similar programs with each user's viewing history. This procedure is implemented for all the programs in the searched list and the programs in the user viewing history. The resulting list of programs is ranked according to the similarity value; the greater the similarity value programs will have the higher priority in the displayed list.

The second list of recommended programs is based on a collaborative filtering approach. This list is based on clustering the users into groups depending on their selected program categories. The number of program categories that the system currently uses is 36 . The clustering operation is done by retrieving the combined viewing history of each user which is saved in the context-aware server and extracting the information related to program categories. Then, the system uses a k-means algorithm to cluster the users into their appropriate groups. Each group includes the most similar users in their selected categories. Then, the system suggests the programs that have been watched by each group and not by the target user.

The third list of recommended programs is based on a contribution from social networks. This list 
includes the movies and TV shows that have been recommended by the users themselves plus the programs that have been recommended by their friends in that network. This is done by creating an application in that particular social network and asking the user to join it in order to benefit from the EPG services. However, after retrieving the information from each social network, the analyzing process is the same. Facebook has been implemented in our EPG system. The user will be asked to sign up in the Facebook part of EPG so that they can login to their Facebook account whenever they want to join the application. Then, the system communicates with the Facebook server to retrieve the required information. The information that is retrieved from each user account is the list of friends who have joined this application and the likes, watched and want to watch movies and TV shows. Facebook provides this information in JSON format, therefore, it is converted into the EPGs internal metadata unified format and analyzed to find the common programs between the user and their friends to rank the programs according to its popularity. Thereafter, the results are saved in the context-aware server and then searched to check the availability of those programs in the content database server as shown in Fig. 2. Details of the missing programs are then sent to the external content sources through the search agents' leader to find them and save them in the content database server. The list of programs will be saved in the context-aware server (in the user matrix). When the user logs in to the EPG, the system will filter this list of programs according to the user's environment.

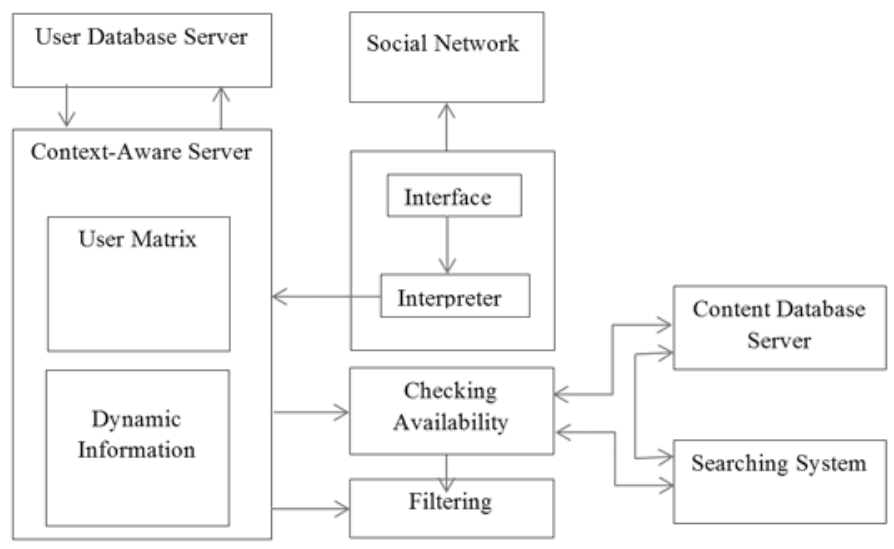

Fig. 2. Social networks contribution.

\subsection{Context-Aware Server}

The context-aware server is responsible of storing the information relevant to each user. This includes user viewing history, user ID and friends' information (for social networks), user preferences and dynamic context information. All of this information will be saved in a matrix which is built by the context-aware manager (see Fig. 1). This matrix represents the user profile which includes all the needed information that will be used in the different operations within the system such as searching operations, recommendations and filtering operation. The viewing history is divided into 24 parts with each representing a particular hour. This will help in defining the user viewing pattern where the viewing time will be taken into account as a factor that affects the recommendation process. The viewing history is updated automatically when the user watches any program. Moreover, the information that has been retrieved from social networks and users' cluster groups will be saved in the user profile (matrix) to use it in the searching and recommendation process. This information is updated every day.

The information retrieval manager retrieves information related to the user device such as device type, resolution and the current network bandwidth. This information will be used in the filtering operation to ensure that the user is provided by a list of programs matching his environment. 


\section{Results and Discussion}

The paper has been focused on how the system is able to access all the available content sources and allow the user to browse his preferences and interesting programs that belongs to different content providers in a single EPG. Additionally, the results showed that the system has personalized the EPG through recommended the programs based on the extracted data from the watched programs, viewing pattern and social networks. This section presents the results that show how our EPG system operates.

\subsection{Retrieving Metadata from Multiple Content Sources}

As we mentioned previously, the system has been connected to BBC-iPlayer, YouTube, Dailymotion and The Movie Database (TMDb) as online content sources in order to verify the ability of the system to communicate with the different content sources. BBC-iPlayer provides its metadata in XML while the others provide their data in JSON. The search agent extracts the metadata from the specific sources and translates this into the EPG internal format. Fig. 3a. shows a program retrieved metadata from one of the sources in JSON format while Fig. 3b. shows this metadata after having been translated. Fig. 3c. shows this metadata after representing each program as a matrix of 22 features in the content database server where all the programs' metadata that retrieved from the different sources are described in that way. Note that the system will insert 'Null' where that particular metadata information is not available.

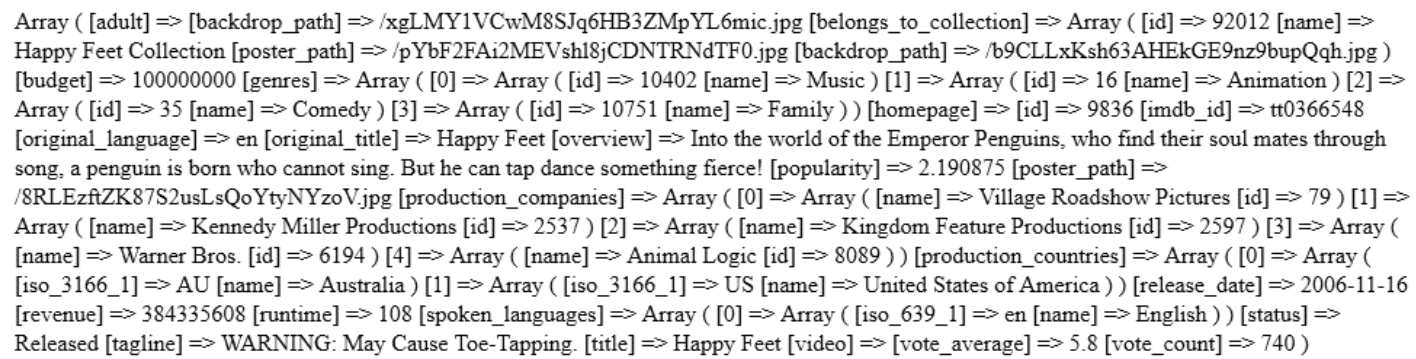

a. Program retrieved information in JSON.

$<$ Title1>Happy Feet</Title1

<Description1>Into the world of the Emperor Penguins, who find their soul mates through song, a penguin is born who cannot sir $<$ Categoryl>Music, Animation, Comedy, Family</Categoryl $<$ Languagel $>$ en $</$ Language $1>$

<ProductionCountry1>Australia, United States of America</ProductionCountryl>

<ProductionCompany $1>V i 1$ lage Roadshow Pictures, Kennedy Mi11er Productions, Kingdom Feature Productions, Warner Bros., Anima 1 L $<$ Rating1 $>2.190875<$ /Rating1>

$<$ Date1 $>2006-11-16</$ Date1>

$<$ Status $1>$ Released $</$ Status $1>$

<Address1>https://www. fan.ty/movies/9836</Address1>

b. Translated program information in XML.

\begin{tabular}{|l|}
\hline \multicolumn{1}{|c|}{215} \\
\hline HAPPY FEET \\
\hline INTO THE WORLD OF THE EMPEROR PENGUINS, WHO FIND THEIR SOUL MATES THROUGH SONG,... \\
\hline MUSIC, ANIMATION, COMEDY, FAMILY \\
\hline Null \\
\hline 2.190875 \\
\hline HAPPY FEET \\
\hline $2006-11-16$ \\
\hline Null \\
\hline EN \\
\hline AUSTRALIA, UNITED STATES OF AMERICA \\
\hline RELEASED \\
\hline Null \\
\hline Null \\
\hline Null \\
\hline Null \\
\hline Null \\
\hline Null \\
\hline Null \\
\hline Null \\
\hline Null \\
\hline Null \\
\hline https://Www.fan.tv/movies/9836 \\
\hline
\end{tabular}

c. Program matrix in the content database server.

Fig. 3. Program metadata stages. 


\subsection{Recommendation Results}

\begin{tabular}{|c|c|c|c|c|c|c|c|}
\hline 11 & 12 & 13 & 14 & 15 & 16 & 17 & 18 \\
\hline $1\langle 22 \times 1$ cell $\rangle$ & $\langle 22 \times 1$ cell $\rangle$ & $\langle 22 \times 1$ cell $\rangle$ & $\langle 22 \times 1$ cell $\rangle$ & $\langle 22 \times 1$ cell $\rangle$ & $\langle 22 \times 1$ cell $\rangle$ & $\langle 22 \times 1$ cell $\rangle$ & $\langle 22 \times 1$ cell $\rangle$ \\
\hline $2\langle 22 \times 1$ cell $\rangle$ & $\langle 22 \times 1$ cell $\rangle$ & $\langle 22 \times 1$ cell $\rangle$ & $\langle 22 \times 1$ cell $\rangle$ & $<22 \times 1$ cell $>$ & ]$<22 \times 1$ cell $\rangle$ & $\langle 22 \times 1$ cell $\rangle$ & $<22 \times 1$ cell $>$ \\
\hline $3\langle 22 \times 1$ cell $\rangle$ & $\langle 22 \times 1$ cell $\rangle$ & $\langle 22 \times 1$ cell $\rangle$ & $\langle 22 \times 1$ cell $\rangle$ & $\langle 22 \times 1$ cell $\rangle$ & $\langle 22 \times 1$ cell $\rangle$ & $\langle 22 \times 1$ cell $\rangle$ & $\langle 22 \times 1$ cell $\rangle$ \\
\hline $4<22 \times 1$ cell $>$ & $\langle 22 \times 1$ cell $\rangle$ & $<22 \times 1$ cell $>$ & $<22 \times 1$ cell $>$ & $\langle 22 \times 1$ cell $>$ & $<22 \times 1$ cell $>$ & $\langle 22 \times 1$ cell $\rangle$ & $\langle 22 \times 1$ cell $\rangle$ \\
\hline $5\langle 22 \times 1$ cell $>$ & $\langle 22 \times 1$ cell $\rangle$ & $<22 \times 1$ cell $>$ & $\langle 22 \times 1$ cell $>$ & [] & $\langle 22 \times 1$ cell $\rangle$ & $\langle 22 \times 1$ cell $\rangle$ & $\langle 22 \times 1$ cell $\rangle$ \\
\hline $6\langle 22 \times 1$ cell $\rangle$ & $\langle 22 \times 1$ cell $\rangle$ & $<22 \times 1$ cell $\rangle$ & $\langle 22 \times 1$ cell $\rangle$ & [] & $\langle 22 \times 1$ cell $\rangle$ & $\langle 22 \times 1$ cell $\rangle$ & $\langle 22 \times 1$ cell $\rangle$ \\
\hline 7 [] & $\langle 22 \times 1$ cell $\rangle$ & $\langle 22 \times 1$ cell $\rangle$ & $\langle 22 \times 1$ cell $\rangle$ & [] & $\langle 22 \times 1$ cell $\rangle$ & $\langle 22 \times 1$ cell $\rangle$ & $\langle 22 \times 1$ cell $\rangle$ \\
\hline 8[] & $\langle 22 \times 1$ cell $\rangle$ & $<22 \times 1$ cell $\rangle$ & $\langle 22 \times 1$ cell $\rangle$ & [] & $\langle 22 \times 1$ cell $\rangle$ & $\langle 22 \times 1$ cell $\rangle$ & [] \\
\hline 9 [] & [] & [] & $\langle 22 \times 1$ cell $>$ & [] & [] & [] & [] \\
\hline 10 [] & [] & [] & $\langle 22 \times 1$ cell $\rangle$ & [] & [] & [] & [] \\
\hline 11 [] & [] & [] & $<22 \times 1$ cell $>$ & [] & [] & [] & [] \\
\hline 12 [] & [] & [] & $<22 \times 1$ cell $>$ & [] & [] & [] & [] \\
\hline 13 [] & [] & [] & $\langle 22 \times 1$ cell $\rangle$ & [] & [] & [] & [] \\
\hline 14 [] & [] & [] & $\langle 22 \times 1$ cell $\rangle$ & [] & [] & [] & [] \\
\hline 15 [] & [] & [] & $<22 \times 1$ cell $\rangle$ & [] & [] & [] & [] \\
\hline
\end{tabular}

Fig. 4. Recommended list for each hour.

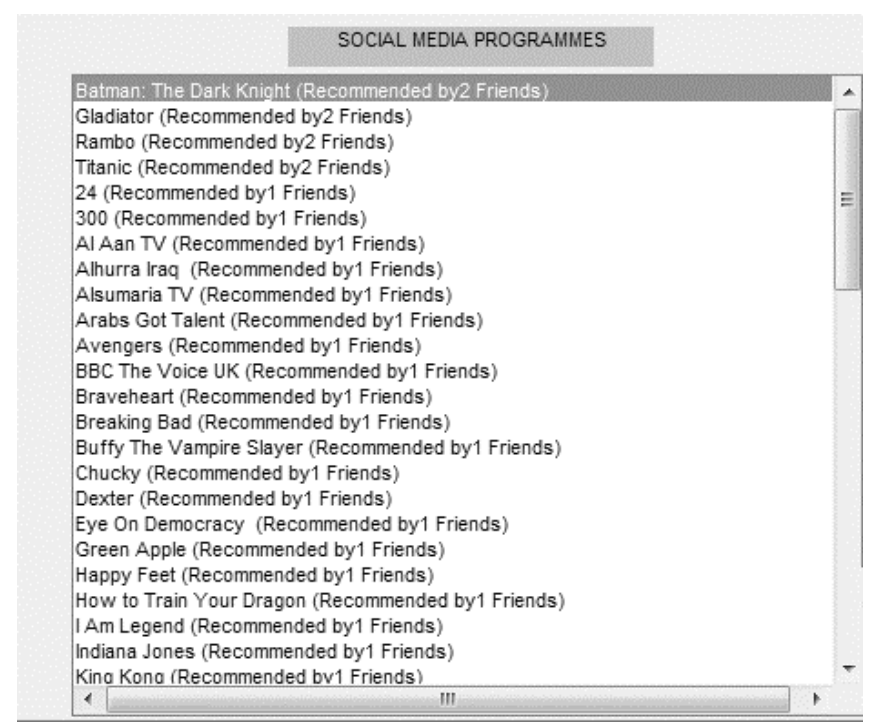

Fig. 5. Recommended list based on facebook contribution.

As mentioned previously, there are three lists of programs that are recommended to the user based on the recommendation approaches that have been used. The first list generated based on the similarity calculations with watched programs in the viewing history. The programs will be recommended based on what the user are get used to watch at each hour and saved in his profile. Fig. 4 Shows the recommended list for a user between $11 \mathrm{am}$ and $6 \mathrm{pm}$, where each column represents one hour of the day. Note that each cell ('22x1' cell) contains a program represented by a matrix of 22 elements (22 program features as shown Fig. $3 c$.$) .$

The second list based on the collaborative filtering approach. K-mean algorithm has been used to cluster the users into appropriate groups based on the categories of the watched programs. We defined 36 categories in our system. Then, the users are divided into groups and the system collects the programs that have been watched by the users who belong to the same group. As a result, a list of programs which includes the collected programs will be recommended to each user except those programs that has been watched by this user themselves. These programs ranked based on the popularity of each program.

The third list has been generated based on integrating Facebook into our system. The application that has been created in Facebook retrieved the required information from the accounts of users who join it. After translating the retrieved information by the interpreter, the system read the XML file and created list of 
programs based on the user preferences and their friends' recommendations. This list has been checked within the content database server and details of the missed programs are sent to the search agent leader to search the different content sources for these programs. Thereafter, the total list of programs saved in the user profile (matrix) and presented to the user when he logged in his EPG. Fig. 5 shows a list of programs for a user depending on the Facebook information.

This list has been ranked based on the number of friends who liked or recommended each program. Moreover, the list has been filtered by removing any program that doesn't match the user dynamic features such as the current available network bandwidth or device resolution.

\subsection{System Evaluation}

As mentioned previously, the key feature of this system is accessing all the available online video content sources in single EPG. Moreover, the system is able to personalize the recommended programs based on the traditional recommendation schemes plus the integration of social networks and user environment features. This section will focus on validating the system to cope with these points.

As we mentioned before, three lists of programs are recommended to each user based on the recommendation techniques used in the system. Each list includes programs retrieved from the different video content sources. Fig. 6 shows the addresses of these programs in one of the recommended list. It is clear from the selected programs' address that they belong to different content sources.

In order to evaluate the recommendation part, we'll discuss two aspects the cold-start problem and recommendation precision. In order to overcome the cold start problem, the registration process for each user in the EPG system includes selecting the categories that the user is interested in (which will be used in users' clustering). Additionally, and in order to provide more interaction with social networks, the user should join the Facebook application to fetch their friends' recommendations. These two features provide recommendations to the user when the user uses the system for the first time. Moreover, this also increases the interaction between friends and family members' selections automatically through the EPG.

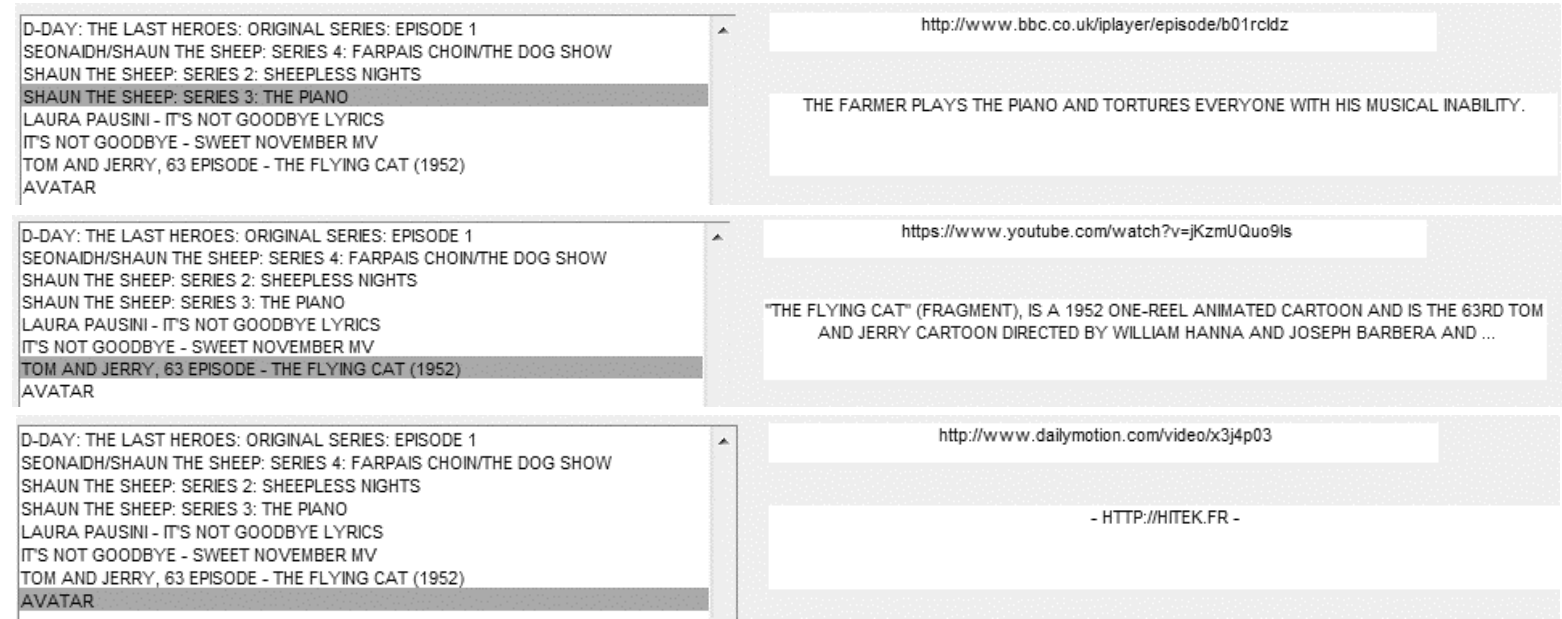

Fig. 6. Part of EPG snapshot showing the program address of recommended list.

On the other hand, the precision of system recommendation has been increased using the social networks contribution. This has been done by asking a group of users to interact with the system to analyse the recommender system performance using the precision as one of the evaluation metrics. The initial results show that the precision has suggested increase 10\% with Facebook contribution as shown in Fig. 7.

Finally, the initial results have been showed that the system able to filter out the programs that don't match the current user environment features such as the user device and network bandwidth. 


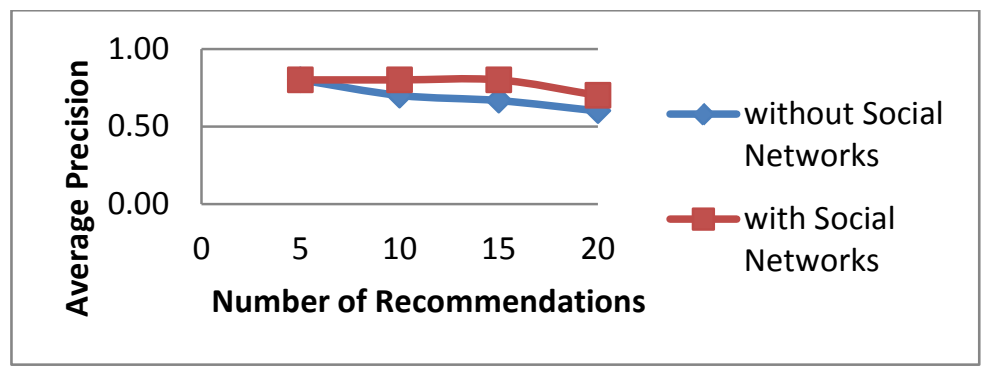

Fig. 7. Average precision with and without facebook contribution.

\section{Conclusions}

Recently, watching television has not been limited to using traditional television set to watch linear broadcasted contents. There is a huge growth in consumption of time-shifted and online content which will start to challenge the future of the traditional television channel as more and more viewers become focused on the programs, rather than the broadcaster or provider who distributes them. Therefore, this new world of online viewing requires a single EPG that is provider agnostic being able to offer the viewer a single point of interface for all content providers.

This paper has presented a new design for an EPG that offers a generic cloud based system which is able to interface to any online content provider. Our EPG takes the program itself as its focus, rather than the provider or source. In addition, the EPG system is able to generate a list of programs that are recommended to watch based on their viewing history, personal preferences and the opinions of family and friends as expressed through social networking thereby achieving a more personalized EPG.

Importantly this new EPG architecture is extensible in that the adoption of an internal metadata format for the system limits the bespoke elements of the design to the search agents that interface to each content provider and modules that interface to each social network environment. These elements can therefore be easily developed to accommodate new providers as they come on line and new social network platforms as they gain in popularity.

In the future we'll implement this system on the other viewing systems of television content such as IPTV with integrating the other content sources such as terrestrial channels and satellite channels besides the online video content sources.

\section{References}

[1] Bondad-Brown, B. A., Rice, R. E., \& Pearce, K. E. (2012). Influences on TV viewing and online user-shared video use: Demographics, generations, contextual age, media use, motivations, and audience activity. Journal of Broadcasting \& Electronic Media, 56(4), 471-493.

[2] Lee, S.-Y., \& Lee, S.-W. (2015). Online video services and other media: Substitutes or complement. Computers in Human Behavior, 51, 293-299.

[3] Oh, H. J., Ozkaya, E., \& LaRose, R. (2014). How does online social networking enhance life satisfaction? The relationships among online supportive interaction, affect, perceived social support, sense of community, and life satisfaction. Computers in Human Behavior, 30, 69-78.

[4] Holanda, P., Guilherme, B., da Silva, A. P. C., \& Goussevskaia, O. (2015). TV Goes Social: Characterizing User Interaction in an Online Social Network for TV Fans. Engineering the Web in the Big Data Era (pp. 182-199). Springer.

[5] Coden A. R., Mak S. W., \& So E. C. (1999). Using multiple search engines to search multimedia data. United State Patent, 5873080. 
[6] Posoldová, A., Oravec, M., \& Rozinaj, G. (2013). Classification and pre-processing of data for television recommendation system. Proceedings of the ELMAR, 2013 55th International Symposium.

[7] Rothschild, L. M. (2011). Systems, devices, and methods for integrated searching and retrieving internet or digital content across a communication network for a multimedia platform. Google Patents.

[8] Kim, M.-E., Cho, J.-M., Yoo, J.-J., Hong, J.-W., \& Kim, S.-H. (2013). A proposal of semantic analysis based integrated multi-level search system for Smart TV. Proceedings of the 15th International Conference on Advanced Communication Technology (ICACT).

[9] Adomavicius, G., \& Tuzhilin, A. (2005). Toward the next generation of recommender systems: A survey of the state-of-the-art and possible extensions. IEEE Transactions on Knowledge and Data Engineering, 17(6), 734-749.

[10] Wu, J., Liu, Q., \& Luo, S. (2008). Clustering technology application in e-commerce recommendation system. Proceedings of the 2008 International Conference on Management of e-Commerce and e-Government.

[11] Barragáns-Martínez, A. B., Costa-Montenegro, E., Burguillo, J. C., Rey-López, M., Mikic-Fonte, F. A., \& Peleteiro, A. (2010). A hybrid content-based and item-based collaborative filtering approach to recommend TV programs enhanced with singular value decomposition. Information Sciences, 180(22), 4290-4311.

[12] Song, S., Moustafa, H., \& Afifi, H. (2012). Advanced IPTV services personalization through context-aware content recommendation. Multimedia, IEEE Transactions on, 14(6), 1528-1537.

[13] Knee, R. A., Reynolds, S. J., Ellis, M. D., \& Hassell, J. G. (2009). Interactive television program guide system for determining user values for demographic categories. Google Patents.

[14] Schaffer, J. D., Lee, K. P., \& Gutta, S. (2011). Three-way media recommendation method and system. Google Patents.

[15] Han, X., Wang, L., Crespi, N., Park, S., \& Cuevas, Á. (2015). Alike people, alike interests? Inferring interest similarity in online social networks. Decision Support Systems, 69, 92-106.

[16] Jiang, M., Cui, P., Liu, R., Yang, Q., Wang, F., Zhu, W., \& Yang, S. (2012). Social contextual recommendation. Proceedings of the 21st ACM International Conference on Information and Knowledge Management.

[17] Shapira, B., Rokach, L., \& Freilikhman, S. (2013). Facebook single and cross domain data for recommendation systems. User Modeling and User-Adapted Interaction, 23(2-3), 211-247.

[18] Quijano-Sanchez, L., Recio-Garcia, J. A., \& Diaz-Agudo, B. (2011). Happymovie: A facebook application for recommending movies to groups. Proceedings of the 23rd IEEE International Conference on Tools with Artificial Intelligence (ICTAI).

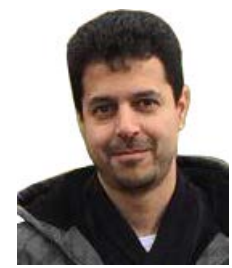

Emad Al-Mohammed was born in 1977. He received his B.Sc. degree in the Department of Electrical Engineering from University of Basrah/Iraq and his M.Sc. degree in the communication engineering in University of Basrah/ Iraq. Currently, he is a Ph.D. student in the Department of Computing, Science and Engineering, University of Salford, UK. His research interests include IPTV, electronic program guide systems and mobile systems.

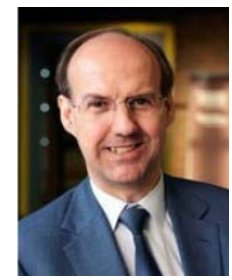

Nigel Linge received his BSc degree in electronics from the University of Salford, UK in 1983, and his PhD in computer networks from the University of Salford, UK, in 1987. He was promoted to professor of telecommunications at the University of Salford, UK in 1997. His research interests include location based and context aware information systems, protocols, mobile systems and the transmission of multimedia applications, including ultra-high definition television 